\title{
Alleviating Cancer Patients' Suffering: Whose Responsibility Is It?
}

\section{By Jorge Grau, PhD}

In medicine, we have historically been better at learning about the body and disease than we have at understanding the human beings who come to us with the ailments. We have acted to relieve pain, consoling patients and families as a complement, but done little to understand and alleviate suffering as a fundamental part of our practice. In fact, only in more recent decades has "suffering" been conceptualized as something apart from pain, associated with distress and its causes. It was Eric T. Cassell, in his ground-breaking work in the 1980 s, who posed the need to consider alleviation of suffering and treatment of illness as twin-and equally important-obligations of the medical profession.

Suffering is defined as a negative, complex emotional and cognitive state, characterized by feeling under constant threat and powerless to confront it, having drained the physical and psychosocial resources that might have made resistance possible. This unique depletion of personal resources is key to understanding suffering.

Cancer patients are particularly vulnerable to suffering, knowing they are confronted with a disease that has long been synonymous with pain, stigmatization, agony and death. For oncologists and other health professionals in the field of cancer treatment, the emotional impact of that knowledge on their patients is particular cause for reflection and action.

Even the suggestion of cancer tends to set off alarms, despite advances in treatment that have transformed many cancers into chronic conditions. In some cultures, like our own, the fear of cancer is so ingrained that at times the diagnosis is hidden from patients, provoked by concern that some will not be able to bear the truth. This can result in extremes-to 'always tell' or 'never tell'-which are not at all helpful. In fact, withholding the truth can be as damaging as always telling the truth in the same way to every person. Deciding if and how to tell a person they have cancer requires assessing each patient's individual circumstances, weighing desired truth against bearable truth.

For any patient, cancer is a minefield of threats. It can incapacitate the body with pain; generate feelings of anger; separate loved ones or alter family roles; require agonizing treatment, hospitalization, and hard decisions; strip patients of their independence; and compromise privacy. Each cancer patient has to face these threats, while continually adjusting to changes in their treatment and prognosis-whether cure, remission, or the prospect of death.

It is imperative that every health professional be fully conscious of and empathize with these realities, committed not only to staving off death or improving the body's performance, but also to prioritizing actions that promote wellbeing and relieve unnecessary suffering.

What does this mean for health systems and cancer care institutions? I believe they must:
- $\quad$ Philosophically incorporate suffering as a unique concept and therapeutic responsibility.

- $\quad$ Adopt an ethical approach that takes into consideration the individual and their unique experiences in the context of their society, culture and family.

- $\quad$ As a result, develop personalized, patient-centered care that addresses suffering.

- $\quad$ Specifically train health professionals to provide care with a sensitive, interdisciplinary, cross-cultural approach that takes into account the patient's intelligence, free will and dignity. Seek to understand the patient and their suffering in a holistic way, recognizing that what is important is how the patient feels, not how the doctor thinks they should feel according to clinical parameters.

- Recognize that suffering may be alleviated, even if it cannot be quantified. Frame this issue so that the overwhelming influence of "measurability" demanded by evidence-based medicine does not relieve us of responsibility to alleviate suffering as much as treat the disease.

- Continually re-examine ethical principles and their application in new circumstances.

...there is no thermometer
to measure suffering...
no pill to relieve it:
the challenge and
responsibility are ours

The aim of caregivers is to alleviate suffering and preserve the best possible quality of life. Suffering's double burden (feeling besieged and impotent combined with diminished resources for fighting back) gives it an essentially subjective quality, at the same time broadening the spectrum for intervention: if the stressors cannot be eliminated or reduced, for example, then we might strengthen the patient's resources to combat them.

We also need viable, ethical strategies to help caregivers know whether they are succeeding or not: has there been a decrease in irritating experiences in provision of services-for example, shorter waiting times for laboratory or clinical test results? Are we supporting appropriate procedures? Have we duly encouraged recreation, occupational therapy and other useful activities that lead to personal fulfillment? Has depression been alleviated? Are we effectively managing fear/hope through competent, empathetic communication? Have the needs of patients and their families been met comprehensively? Do patients give greater meaning to their lives and value to their accomplishments?

Just as there is no thermometer to measure suffering, there is no pill to relieve it: the challenge and responsibility are ours. Addressing suffering depends upon professionals, institutions and health systems making alleviation of suffering a vital goal of their practice and not simply a testament to humanistic patient relations or the personification of the "good doctor". Suffering is intrinsic to protracted illnesses such as cancer, not merely a corollary. Relief of suffering must be made an essential component of first-line therapy. -1 - 